\title{
Search for long-lived particles at LHCb
}

\author{
Alberto Lusiani* ${ }^{\dagger}$ \\ Scuola Normale Superiore and INFN, sezione di Pisa \\ E-mail: alberto.lusiani@pi.infn.it
}

Three searches are presented for long-lived particles with specific production and decay features: pairs of long-lived particles from Higgs boson decay and decaying either to jet pairs or to three quarks, and long-lived particles decaying semileptonically. The searches are based on protonproton collision data corresponding to integrated luminosities up to $3.0 \mathrm{fb}^{-1}$, collected by the $\mathrm{LHCb}$ detector at centre-of-mass energies of 7 and $8 \mathrm{TeV}$, using data sub-samples taken with suitable triggers. All three searches observed no excess above background and set limits on the production cross-sections as a function of the mass and lifetime of the long-lived particles.

The European Physical Society Conference on High Energy Physics

5-12 July, 2017

Venice

* Speaker.

${ }^{\dagger}$ On behalf of the LHCb collaboration. 


\section{Introduction}

Several New Physics models predict particles that are long-lived, typically because they are loosely coupled to the Standard Model (SM) fields.

The LHCb detector [1] at the Large Hadron Collider (LHC) at CERN is a forward spectrometer that has been designed for precision measurements of $\mathrm{CP}$ violation and for studies of rare heavy flavour decays, but can also be used reliably for a variety of New Physics searches. Regarding hypothetical long-lived particles (LLPs), LHCb is effective for detecting and identifying particles with a lifetime short enough to decay inside the LHCb detector and long enough to decay at a significant radial displacement from the beams. This corresponds to lifetimes of the order of $1 \mathrm{ps,}$ which implies that such particles have narrow widths. A secondary vertex with a significant displacement from the beam axis is important to suppress backgrounds from SM processes originating on the primary interaction vertex. Vertices up to $20 \mathrm{~cm}$ downstream of the interaction region are fitted using tracks reconstructed from hits in the Vertex Locator (VELO) and can fire the experiment trigger. Vertices farther away than $20 \mathrm{~cm}$ and up to $200 \mathrm{~cm}$ along the beam line are fitted using tracks reconstructed with relatively worse resolution by the downstream LHCb subdetectors, and don't contribute to the trigger.

The main backgrounds for LLP searches at LHCb are tracks coming from displaced vertices corresponding to either heavy flavour decays or secondary interactions with the detector. To suppress these backgrounds, we veto events with either vertices reconstructed as inside the detector material or LLP masses measured to be consistent with known SM particles.

\section{Search for long-lived particles decaying to jet pairs}

Models with a hidden-sector non-Abelian gauge group that couple to the SM via a Higgs boson portal may include long-lived bosons (named hidden-valley pion or $\pi_{\mathrm{v}}$ ) that can be pair-produced in the decay of the SM Higgs [2, 3, 4, 5, 6], and then decay to two hadronic jets. In simulated events, often only one of the two $\pi_{\mathrm{v}}$ decays occurs inside the LHCb acceptance. Therefore, to maximize efficiency, we search for evidence of at least one LLP candidate, consisting of two hadronic jets originating from a displaced vertex.

We use data collected with appropriated triggers for this search from $p p$ collisions at centre-ofmass energy of $\sqrt{s}=7 \mathrm{TeV}$ in 2011 (with an integrated luminosity of $0.62 \mathrm{fb}^{-1}$ ) and at $\sqrt{s}=8 \mathrm{TeV}$ in $2012\left(1.38 \mathrm{fb}^{-1}\right)$. This analysis updates an earlier one based on just the 2011 dataset [7].

We search for two hadronic jets consistent with coming from a vertex whose distance from the beam in the transverse plane is $R_{x y}>0.4 \mathrm{~mm}$. The di-jet momentum must be aligned with the displacement from one reconstructed primary vertex to the candidate vertex. The two jets must have a small separation to suppress background from back-to-back SM jets. We find no evidence for a signal over the expected background in the di-jet invariant mass distribution and we set appropriate upper limits [8] (Fig. 1) for the production cross-section $\left(\sigma / \sigma_{g g \rightarrow H^{0}}^{S M}\right) \cdot \mathscr{B}\left(H^{0} \rightarrow \pi_{\mathrm{v}} \pi_{\mathrm{v}}\right)$, where $\sigma$ is the observed cross-section, $\sigma_{g g \rightarrow H^{0}}^{S M}$ is the SM Higgs boson production cross-section via the gluon fusion process and $\mathscr{B}\left(H^{0} \rightarrow \pi_{\mathrm{v}} \pi_{\mathrm{v}}\right)$ is the branching fraction of the Higgs boson decay to $\pi_{\mathrm{v}}$ particles. 


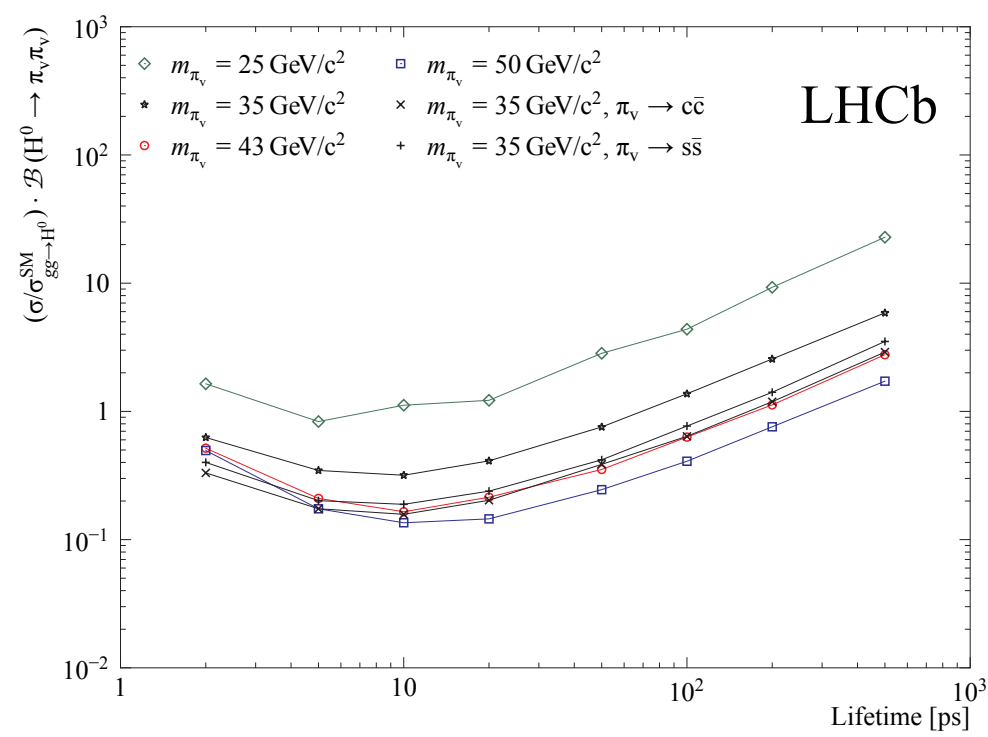

Figure 1: Observed 95\%-confidence-level upper limit on the production cross section of $\pi_{\mathrm{v}}$ pairs as a function of their lifetime and for different masses and decay modes.

\section{Search for long-lived particles decaying to three quarks}

Certain minimal supergravity (mSUGRA) models $[9,10]$ predict Higgs-like bosons that can be produced in $p p$ collisions and decay to two LLPs neutralinos subsequently decaying to three quarks. This latter decay is mediated by a very weak baryon-number-violating interaction [11]. We test this prediction by searching for candidate events containing two high multiplicity jets that are consisting with originating from two separate vertices that also have a significant displacement from the beam axis.

We search for two jets with at least four tracks each, in $0.62 \mathrm{fb}^{-1}$ of data collected in 2011 with appropriate triggers. One jet candidate must have a minimum invariant mass larger than $3.5 \mathrm{GeV} / c^{2}$, and the other larger than $4.5 \mathrm{GeV} / c^{2}$.

We find no evidence for a signal peak over the expected background in the reconstructed invariant mass distribution and we set the limits [12] summarized in Figure 2.

\section{Search for massive long-lived particles decaying to a muon and two jets}

Some mSUGRA models with R-parity violation predict massive long-lived neutralinos that decay to a muon and two jets $[13,10]$, via a very weak R-parity-violating interaction. We test these model predictions by searching for candidate events containing a LLP with mass in the range $20-193 \mathrm{GeV} / \mathrm{c}^{2}$ and lifetime 5-100 ps. We consider several specific models for the production of one or two LLPs in the same event, and we also consider a full inclusive search for LLP production as simulated by PYTHIA 6 [14] by taking into account a full set of MSSM/mSUGRA production mechanisms.

In the data collected in $2011\left(1 \mathrm{fb}^{-1}\right)$ and $2012\left(2 \mathrm{fb}^{-1}\right)$, we search for an isolated muon and at least 4 tracks consistent with originating from a common vertex with significant displacement from 

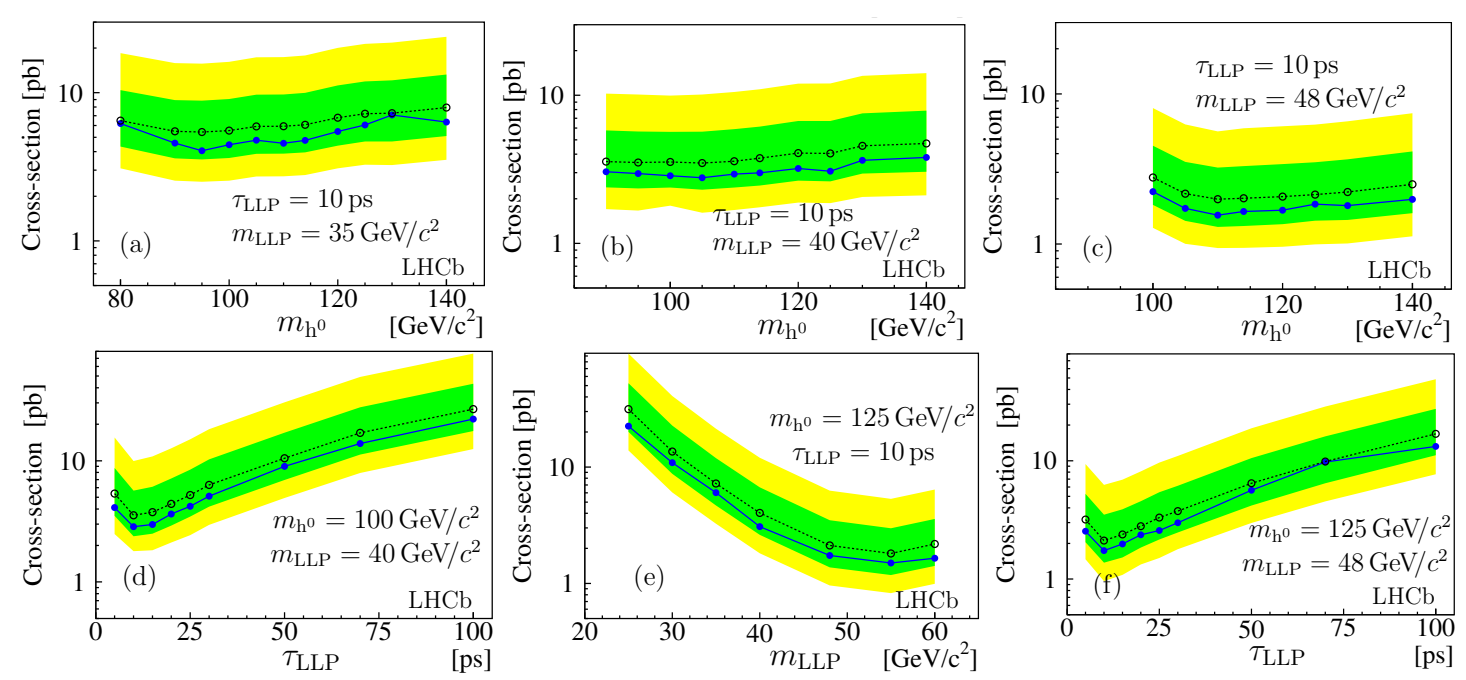

Figure 2: Expected (open dots with $1 \sigma$ and $2 \sigma$ bands) and observed (full dots) upper limits at $95 \%$ confidence level, for different Higgs-like-boson masses [(a) - (c)], for different LLP lifetimes [(d) and (f) ], and as a function of the LLP mass [(e)]. The values of the other parameters are reported on the plots.

the beam axis. The muon is required to have a transverse momentum larger than $12 \mathrm{GeV} / c$. We search for a peak over the estimated background in the recostructed LLP invariant mass distribution. We find no significant signal and we set limits [15] for all models considered and separately for the $2011(7 \mathrm{TeV})$ and $2012(8 \mathrm{TeV})$ datasets. Figure 3 hows examples of these limits.

\section{Conclusion}

LHCb found no statistically significant excess of long-lived particles when considering three different models of production and decay modes. When comparing published results on searches for LLPs decaying to two jets, LHCb is complementary to ATLAS and CMS and is more sensitive to shorter lifetimes $(<1 \mathrm{ps})$ and lighter masses [16].

\section{References}

[1] LHCb collaboration, R. Aaij et al., LHCb Detector Performance, Int. J. Mod. Phys. A30 (2015) 1530022, [1412.6352].

[2] M. J. Strassler and K. M. Zurek, Echoes of a hidden valley at hadron colliders, Phys. Lett. B651 (2007) 374-379, [hep-ph/ 0604261$].$

[3] M. J. Strassler and K. M. Zurek, Discovering the Higgs through highly-displaced vertices, Phys. Lett. B661 (2008) 263-267, [hep-ph/ 0605193$].$

[4] S. Chang, R. Dermisek, J. F. Gunion and N. Weiner, Nonstandard Higgs Boson Decays, Ann. Rev. Nucl. Part. Sci. 58 (2008) 75-98, [0801.4554].

[5] N. Craig, A. Katz, M. Strassler and R. Sundrum, Naturalness in the Dark at the LHC, JHEP 07 (2015) 105, [1501.05310].

[6] D. Curtin and C. B. Verhaaren, Discovering Uncolored Naturalness in Exotic Higgs Decays, JHEP 12 (2015) 072, [1506.06141]. 

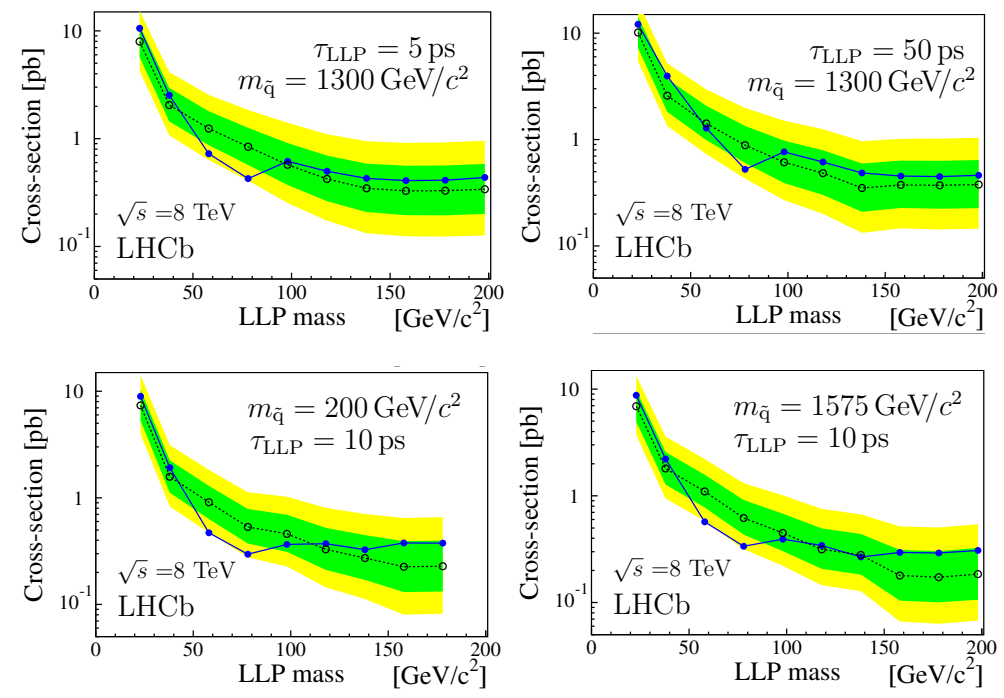

Figure 3: Expected (open dots with $1 \sigma$ and $2 \sigma$ bands) and observed (full dots) cross-section times branching fraction upper limits at $95 \%$ confidence level, as a function of the LLP mass from the $2012(8 \mathrm{TeV})$ dataset. The theoretical models assume the full set of SUSY production processes available in PYTHIA 6 with default parameter settings. The four plots report limits for different choices of the squark mass and the LLP lifetime. The gluino mass is set to $2000 \mathrm{GeV} / c^{2}$.

[7] LHCb COllaboration, R. Aaij et al., Search for long-lived particles decaying to jet pairs, Eur. Phys. J. C75 (2015) 152 LHCb-PAPER-2014-062, CERN-PH-EP-2014-291, [1412 . 3021 ].

[8] LHCB COllaboration, R. Aaij et al., Search for long-lived particles decaying to jet pairs in LHCb Run 1 data, LHCb-PAPER-2016-065.

[9] D. E. Kaplan and K. Rehermann, Proposal for Higgs and superpartner searches at the LHCb experiment, JHEP 10 (2007) 056, [0 705.3426].

[10] P. W. Graham, D. E. Kaplan, S. Rajendran and P. Saraswat, Displaced Supersymmetry, JHEP 07 (2012) 149, [1204.6038].

[11] L. M. Carpenter, D. E. Kaplan and E.-J. Rhee, Reduced fine-tuning in supersymmetry with R-parity violation, Phys. Rev. Lett. 99 (2007) 211801, [hep-ph/ 0607204 ].

[12] LHCb Collaboration, R. Aaij et al., Search for Higgs-like bosons decaying into long-lived exotic particles, Eur. Phys. J. C76 (2016) 664 LHCb-PAPER-2016-014, CERN-EP-2016-188, [1609.03124].

[13] B. C. Allanach, A. Dedes and H. K. Dreiner, R parity violating minimal supergravity model, Phys. Rev. D69 (2004) 115002, [hep-ph/ 0309196$].$

[14] T. Sjostrand, S. Mrenna and P. Z. Skands, PYTHIA 6.4 Physics and Manual, JHEP 05 (2006) 026, [hep-ph/0603175].

[15] LHCb COllaboration, R. Aaij et al., Search for massive long-lived particles decaying semileptonically in the LHCb detector, Eur. Phys. J. C77 (2017) 224 LHCb-PAPER-2016-047, CERN-EP-2016-283, [1612.00945].

[16] P. N. Y. David, Search for Exotic Long-Lived Particles with the LHCb Detector. PhD thesis, Vrije U., Amsterdam, 2016. 